\title{
THE FREDHOLM SPECTRUM OF THE SUM AND PRODUCT OF TWO OPERATORS
}

BY

\section{J ACK SHAPIRO AND MORRIS SNOW( 1 )}

ABSTRACT. Let $C(X)$ denote the set of closed operators with dense domain on a Banach space $X$, and $L(X)$ the set of all bounded linear operators on $X$. Let $\Phi(X)$ denote the set of all Fredholm operators on $X$, and $\sigma_{\phi}(A)$ the set of all complex numbers $\lambda$ such that $(\lambda-A) \notin(X)$. In this paper we establish conditions under which $\sigma_{\phi}(A+B) \subseteq \sigma_{\phi}(A)+\sigma_{\phi}(B), \sigma_{\phi}(\overline{B A}) \subseteq \sigma_{\phi}(A) \cdot \sigma_{\phi}(B)$, and $\sigma_{\phi}(A B) \subseteq \sigma_{\phi}(A) \sigma_{\phi}(B)$.

In this paper we will use the operational calculus developed in [3] to establish a property of the Fredholm spectrum of the sum and product of two operators.

Definition 1. A closed operator $A$ from a Banach space $X$ to a Banach space $Y$ is called a Fredholm operator if:

(1) the domain of $A, D(A)$, is dense in $X$.

(2) $\alpha(A)=\operatorname{dim}[N(A)]<\infty$.

(3) $R(A)$, the range of $A$, is closed in $Y$.

(4) $\beta(A)$, the codimension of $R(A)$ in $Y$, is finite.

It is shown in [1, Lemma 332] that condition (4) implies condition (3). A discussion of Fredholm operators can be found in [2].

We denote the set of Fredholm operators from $X$ to $Y$ by $\Phi(X)$.

Definition 2. $\lambda \in \Phi_{A}$ if and only if $(\lambda-A) \in \Phi(X)$.

Definition 3. $\lambda \in \sigma_{\phi}(A)$ if and only if $\lambda \notin \Phi_{A}$.

Definition 4. A bounded operator $B$ will be called a quasi-inverse of the closed operator $A$ if:

(1) $R(B) \subset D(A)$ and $A B=I+K_{1}, K_{1} \in \mathcal{K}(X)$.

(2) $B A=I+K_{2}, K_{2} \in \mathcal{K}(X)$.

$K(X)$ denotes the set of all compact operators on $X$.

Presented to the Society, May 5, 1972; received by the editors June 11, 1973 and, in revised form, August 7, 1973.

AMS (MOS) subject classifications (1970). Primary 47B30; Secondary 47 A60, $47 \mathrm{~B} 05$.

Key words and phrases. Fredholm operator, Fredholm spectrum, operational calculus.

(1) This research was partially funded by a Faculty Research Award Program grant, from the City University of New York.

Copyright $\odot$ 1974, American Mathematical Society 
By [2, Theorem 2.9], $\Phi_{A}$ is open and is thus the union of a disjoint collection of connected open sets. Each such set, $\Phi_{i}(A)$, will be called a component of $\Phi_{A}$.

Let $C(X)$ denote the set of closed operators on $X$ with dense domain.

Suppose $A \in C(X)$ with $\Phi_{A}$ not empty, and let $\lambda \in \Phi_{A}$. In [3], a quasiinverse of $\lambda-A, R_{\lambda}^{\prime}(A)$, was constructed in the following way. In each $\Phi_{i}(A)$, a fixed point, $\lambda_{i}$, is chosen in a prescribed manner. There exist subspaces, $X_{i}$ and $Y_{i}$ such that $X=i\left(\lambda_{i}-A\right) \oplus X_{i}, X_{i}$ is closed, and $X=Y_{i} \oplus R\left(\lambda_{i}-A\right)$, $\operatorname{dim} Y_{i}=\beta\left(\lambda_{i}-A\right)$.

Let $F_{1 i}$ be the projection of $X$ onto $N\left(\lambda_{i}-A\right)$ along $X_{i}$, and let $F_{2 i}$ be the projection of $X$ onto $Y_{i}$ along $R\left(\lambda_{i}-A\right) . F_{1 i}$ and $F_{2 i}$ are bounded finite rank operators. $\left.\left(\lambda_{i}-A\right)\right|_{D(A) \cap X_{i}}$ has a bounded inverse, $A_{i} ; A_{i}: R\left(\lambda_{i}-A\right) \stackrel{\text { onto }}{\longrightarrow} D(A) \cap X_{i}$.

Let the operator $T_{i}$ be defined by: $T_{i} x=A_{i}\left(I-F_{2 i}\right) x . T_{i}$ is a quasi-inverse of $\left(\lambda_{i}-A\right)$.

$R_{\lambda}^{\prime}(A)$ is then defined by $R_{\lambda}^{\prime}(A)=T_{i}\left[\left(\lambda-\lambda_{i}\right) T_{i}+I\right]^{-1}$ when $\lambda \in \Phi_{i}(A)$ and $-1 /\left(\lambda-\lambda_{i}\right) \in \rho\left(T_{i}\right)$. In [3, Theorems 2 and 5, $\left.\$ 2\right], R_{\lambda}^{\prime}(A)$ is shown to be a quasiinverse of $(\lambda-A)$ defined and analytic for all $\lambda \in \Phi_{A}$ except for at most an isolated set, $\Phi^{0}(A)$, having no accumulation point in $\Phi_{A}$.

Lemma 1.1. Let $n$ be a positive integer and $A \in C(X)$ sucb that $\Phi_{A}$ is not empty. Then for eacb $\lambda \in\left\{\Phi_{A} \backslash \Phi^{0}(A)\right\}$, there exists a subspace $V_{\lambda}$, dense in $X$ and depending on $\lambda$, such that $\forall x \in V_{\lambda}, R_{\lambda}^{\prime}(A) x \in D\left(A^{n}\right)$.

Proof. Let $\lambda \in\left\{\Phi_{A} \backslash \Phi^{0}(A)\right\}$. By [2, Theorem 2.5], $D\left(A^{n}\right)=D\left[(\lambda-A)^{n}\right]$ is dense in $X$ for all $n$. Therefore, $T_{i}^{-1}\left[D\left(A^{n}\right)\right] \cap R(A)=D\left(A^{n-1}\right) \cap R(A)$ is dense in $R(A)$. By $T_{i}^{-1}\left[D\left(A^{n}\right)\right]$ we mean $\left\{x \mid T_{i} x \in D\left(A^{n}\right)\right\}$. Let $Y_{i}$ be the complement of $R(A)$ used in the construction of $T_{i}$. Since $T_{i}: Y_{i} \rightarrow 0 \in D\left(A^{n}\right)$ and $X=$ $R(A)+Y_{i}$, we have $T_{i}^{-1}\left[D\left(A^{n}\right)\right]=\left\{T_{i}^{-1}\left[D\left(A^{n}\right)\right] \cap R(A)\right\} \oplus Y_{i}$ is dense in $X$. Therefore, $V_{\lambda}=\left[\left(\lambda-\lambda_{i}\right) T_{i}+I\right]\left\{T_{i}^{-1}\left[D\left(A^{n}\right)\right]\right\}$ is dense in $X$ because $\left[\left(\lambda-\lambda_{i}\right) T_{i}+I\right]$ is invertible. Q.E.D.

We denote the set of all bounded operators on $X$ by $L(X)$.

Lemma 1.2. Let $A \in \Phi(X), B \in L(X)$, and $K \in K(X)$. Suppose $\left.A B\right|_{V}=\left.K\right|_{V}$ where $V$ is a dense subspace of $X$. Then $B \in K(X)$.

Proof. There exists $A_{0} \in L(X)$ such that $A_{0} A=I-K_{1}, K_{1} \in \mathcal{K}(X)$.

$$
\left.A_{0} A B\right|_{V}=\left.A_{0} K\right|_{V},\left.\quad\left(I-K_{1}\right) B\right|_{V}=\left.K_{2}\right|_{V},\left.\quad B\right|_{V}=\left.\left(K_{1} B+K_{2}\right)\right|_{V} .
$$

Since $\&$ and $\left(K_{1} B+K_{2}\right)$ are bounded, and $V$ is dense, we have $B=K_{1} B+K_{2}$ by contınuity. Q.E.D.

Lemma 1.3. Let $B \in L(X), A \in C(X), \mu \in\left\{\Phi_{B} \backslash \Phi^{0}(B)\right\}$ and $\lambda \in\left\{\Phi_{A} \backslash \Phi^{0}(A)\right\}$. Let there exist a positive integer $n$ and a compact operator $K_{1}$, sucb that 
$B: D\left(A^{n}\right) \rightarrow D(A)$ and $A B x=B A x+K_{1} x, \forall x \in D\left(A^{n}\right)$. Then there exists a compact operator $K$, depending analytically on $\lambda$ and $\mu$, sucb that

$$
R_{\lambda}^{\prime}(A) R_{\mu}^{\prime}(B)=R_{\mu}^{\prime}(B) R_{\lambda}^{\prime}(A)+K .
$$

Proof. By Lemma 1.1 there exists a subspace $V_{\lambda}$, dense in $X$, such that $\forall x \in V_{\lambda}, R_{\lambda}^{\prime}(A)_{x} \in D\left(A^{n}\right)$. Let $x \in V_{\lambda}$.

$$
\begin{aligned}
(\lambda-A) B R_{\lambda}^{\prime}(A) x & =\left[B(\lambda-A)-K_{1}\right] R_{\lambda}^{\prime}(A) x \\
& =\left[B\left(I-K_{2}\right)+K_{3}\right] x \\
& =B x+K_{4} x ; \\
(\lambda-A) R_{\lambda}^{\prime}(A) B x & =\left(I-K_{2}\right) B x=B x-K_{5} x .
\end{aligned}
$$

Therefore, $(\lambda-A)\left[B R_{\lambda}^{\prime}(A)-R_{\lambda}^{\prime}(A) B\right] x=K_{6} x$. Since this equality holds for all $x \in V_{\lambda}$, we have by Lemma 1.2 , that $B R_{\lambda}^{\prime}(A)-R_{\lambda}^{\prime}(A) B_{\lambda}=K^{\prime}$, and $B R_{\lambda}^{\prime}(A)=$ $R_{\lambda}^{\prime}(A) B+K^{\prime}, K^{\prime}, K_{i} \in K(X)$ for $i=1,2, \cdots, 6$.

$$
\begin{aligned}
(\mu-B)\left[R_{\mu}^{\prime}(B) R_{\lambda}^{\prime}(A)-R_{\lambda}^{\prime}(A) R_{\mu}^{\prime}(B)\right] & =\left(I-K_{7}\right) R_{\lambda}^{\prime}(A)-(\mu-B) R_{\lambda}^{\prime}(A) R_{\mu}^{\prime}(B) \\
& =R_{\lambda}^{\prime}(A)+K_{8}-R_{\lambda}^{\prime}(A)\left(I-K_{7}\right)+K_{9}=K_{10} .
\end{aligned}
$$

Therefore, $R_{\mu}^{\prime}(B) R_{\lambda}^{\prime}(A)-R_{\lambda}^{\prime}(A) R_{\mu}^{\prime}(B)=K$, and $R_{\mu}^{\prime}(B) R_{\lambda}^{\prime}(A)=R_{\lambda}^{\prime}(A) R_{\mu}^{\prime}(B)+K$, $K, K_{i} \in \mathcal{K}(X)$ for $i=7,8,9,10$. The analyticity of $K$ in $\lambda$ and $\mu$ follows from the analyticity of $R_{\mu}^{\prime}(B)$ and $R_{\lambda}^{\prime}(A)$.

Theorem 1. Let $B \in L(X)$ and $A \in C(X)$. Suppose there exist a positive integer $n$ and a compact operator $K$ sucb that $B: D\left(A^{n}\right) \rightarrow D(A)$ and $B A x=A B x$ $+K x$ for all $x \in D\left(A^{n}\right)$. Then $\sigma_{\phi}(A+B) \subseteq \sigma_{\phi}(A)+\sigma_{\phi}(B)$. If $\sigma_{\phi}(A)$ is empty, we interpret $\sigma_{\phi}(A)+\sigma_{\Phi}(B)$ to be the empty set.

Proof. If $\sigma_{\phi}(A)+\sigma_{\phi}(B)$ is the entire complex plane, then the theorem is trivially ture. We therefore assume that $\sigma_{\phi}(A)+\sigma_{\phi}(B)$ is not the entire plane.

Let $\gamma$ be a fixed point not contained in $\sigma_{\phi}(A)+\sigma_{\phi}(B)$. We shall show that $\gamma \in \Phi_{(A+B)^{\circ}}$. If $\lambda \in \sigma_{\phi}(B)$, then $(\gamma-\lambda) \in \Phi_{A}$. Since $\sigma_{\phi}(A)$ is closed and $\sigma_{\phi}(B)$ is compact, there exists an open set $U \supset \sigma_{\phi}(B)$ such that $B(U)$, the boundary of $U$, is bounded, and when $\lambda \in U,(\gamma-\lambda) \in \Phi_{A^{*}}$. Let $A_{1}=\gamma-A_{\bullet}(\gamma-\lambda) \in \Phi_{A}$ if and only if $\lambda \in \Phi_{A_{1}}$. Therefore, $\sigma_{\Phi}(B) \subset U \subset \Phi_{A_{1}}$. There exists a bounded Cauchy domain $D$ such that $\sigma_{\Phi}(B) \subset D \subset U$. See [4, Theorem 3.3]. Since $\Phi^{0}\left(A_{1}\right)$ does not accumulate in $\Phi_{A_{1}}$ and $\Phi^{0}(B)$ does not accumulate in $\Phi_{B}, D$ can be chosen so that $R_{\lambda}^{\prime}\left(A_{1}\right)$ and $R_{\lambda}^{\prime}(B)$ are analytic on $B(D)$, the boundary of $D$.

Define the operators $S_{1}$ and $S_{2}$ by

$$
S_{1}=\frac{-1}{2 \pi i} \int_{+B(D)} R_{\lambda}^{\prime}\left(A_{1}\right) R_{\lambda}^{\prime}(B) d \lambda
$$


and

$$
S_{2}=\frac{-1}{2 \pi i} \int_{+B(D)} R_{\lambda}^{\prime}(B) R_{\lambda}^{\prime}\left(A_{1}\right) d \lambda
$$

$R_{\lambda}^{\prime}\left(A_{1}\right)$ is of the form $T C(\lambda)$, where $C(\lambda)$ is bounded operator valued analytic function of $\lambda$ and $T$ is a fixed bounded operator such that $T: X \rightarrow D\left(A_{1}\right)=D(A)$.

Therefore, $S_{1}: X \rightarrow D(A)$.

We will now show that there exist compact operators $K_{1}$ and $K_{2}$ such that $(\gamma-B-A) S_{1}=I+K_{1}$ and $S_{2}(\gamma-B-A)=\left.\left(I+K_{2}\right)\right|_{D(A)}$.

$$
\begin{aligned}
\gamma-B-A & =(\gamma-\lambda-A)+(\lambda-B)=-\left(\lambda-A_{1}\right)+(\lambda-B) . \\
(\gamma-B-A) S_{1} & =\frac{-1}{2 \pi i} \int_{+B(D)}-\left(\lambda-A_{1}\right) R_{\lambda}^{\prime}\left(A_{1}\right) R_{\lambda}^{\prime}(B) d \lambda \\
& =\frac{-1}{2 \pi i} \int_{+B(D)}(\lambda-B) R_{\lambda}^{\prime}\left(A_{1}\right) R_{\lambda}^{\prime}(B) d \lambda .
\end{aligned}
$$

Since $\left(\lambda-A_{1}\right) R_{\lambda}^{\prime}\left(A_{1}\right)$ is of the form $I+F(\lambda)$ where $F(\lambda)$ is a bounded finite rank operator depending analytically on $\lambda$ and $(2 \pi i)^{-1} \int_{+B(D)} R_{\lambda}^{\prime}(B) d \lambda$ is of the form $I+K_{3}$, see [3, Theorem $\left.13, \$ 2\right]$, the first integral is of the form $I+K_{4}$. $K_{3}, K_{4} \in \mathcal{K}(X)$.

By Lemma 1.3, there exists a compact operator $K(\lambda)$, depending analytically on $\lambda$, such that $R_{\lambda}^{\prime}\left(A_{1}\right) R_{\lambda}^{\prime}(B)=R_{\lambda}^{\prime}(B) R_{\lambda}^{\prime}\left(A_{1}\right)+K(\lambda)$. Therefore, the second integral is equal to

$$
\frac{-1}{2 \pi i} \int_{+B(D)}(\lambda-B) R_{\lambda}^{\prime}(B) R_{\lambda}^{\prime}\left(A_{1}\right) d \lambda-\frac{1}{2 \pi i} \int_{+B(D)}(\lambda-B) K(\lambda) d \lambda
$$

The first of these two integrals equals $\int_{+B(D)} R_{\lambda}^{\prime}\left(A_{1}\right) d \lambda+K_{5}, K_{5} \in \mathcal{K}(X)$. As in [3, proof of Theorem 10, $\S 2], \int_{+B(D)} R_{\lambda}^{\prime}(A) d \lambda$ is compact. Since $(2 \pi i)^{-1} \int_{+B(D)}(\lambda-B) K(\lambda) d \lambda$ is also compact, we have that $(\gamma-B-A) S_{1}=I+K_{1}$, $K_{1} \in \mathcal{K}(X)$.

By a similar argument we have that $S_{2}(\gamma-B-A)=I+K_{2}, K_{2} \in K(X)$. Therefore, by [2, Lemma 2.4], $(\gamma-B-A) \in \Phi(X)$ and, thus, $\gamma \in \Phi_{A+B^{\circ}}$. Therefore, $\sigma_{\Phi}(A+B) \subseteq \sigma_{\Phi}(A)+\sigma_{\Phi}(B)$. This completes the proof of the theorem.

Theorem 2. Let $A \in C(X)$ and $B \in L(X) \cap \Phi(X)$. Let $B: D(A) \rightarrow D(A)$ and let there exist a compact operator $K$ such that $B A x=A B x+K x, \forall x \in D(A)$. Then $B A$ is preclosed and

(1) $\sigma_{\phi}(\overline{B A}) \subseteq \sigma_{\phi}(A) \sigma_{\phi}(B)$, and

(2) $\sigma_{\phi}(A B) \subseteq \sigma_{\phi}(A) \sigma_{\Phi}(B)$,

Proof. Since $\left.(A B)\right|_{D(A)}$ is preclosed and $K$ is bounded, we have that $B A$ is preclosed.

Let $\mathrm{C}$ denote the set of all complex numbers. Since $0 \notin \sigma_{\phi}(B)$ and $\sigma_{\phi}(B)$ 
is not empty, we have that if $\sigma_{\phi}(A)=\mathrm{C}$ then $\sigma_{\phi}(B) \sigma_{\phi}(A)=\mathrm{C}$, and the lemma is established. We therefore assume that $\sigma_{\phi}(A) \neq \mathrm{C}$.

Suppose $\gamma$ is a fixed point not in $\sigma_{\phi}(B) \sigma_{\phi}(A)$. We proceed to show that $\gamma \in \Phi(\overline{B A})$.

Since $\sigma_{\Phi}(A)$ is closed, $\sigma_{\Phi}(B)$ is compact, and $0 \notin \sigma_{\Phi}(B)$, there exists an open set $U \supset \sigma_{\phi}(B)$ such that $0 \notin U, B(U)$, the boundary of $U$, is bounded, and $(\gamma-\mu A) \in \Phi(X) \forall \mu \in U$. Let $D$ be a bounded Cauchy domain such that $\sigma_{\phi}(B) \subset$ $D \subseteq U . \quad(\gamma-\mu A)=\mu \gamma(1 / \mu-(1 / \gamma) A)=(\gamma / \lambda)(\lambda-(1 / \gamma) A)$, where $\lambda=1 / \mu$.

Let $D^{\prime}$ be the image of $D$ under the map $\lambda=1 / \mu$. Let $A_{1}=(1 / \gamma) A$. Since $\forall \mu \in \bar{D}, 1 / \mu \in \Phi_{A_{1}}$, and since $R_{\lambda}^{\prime}\left(A_{1}\right)$ is analytic in $\lambda$ throughout $\Phi_{A_{1}}$, except for at most an isolated set having no accomulation point in $\Phi_{A_{1}}$, we can assume that $R_{\lambda}^{\prime}\left(A_{1}\right)$ is analytic in $\lambda$ on $B\left(D^{\prime}\right)$. Note. $R_{\lambda}^{\prime}\left(A_{1}\right)$ is of the form $T C(\lambda)$ where $C(\lambda)$ is an analytic operator valued function of $\lambda$ and $T$ is a bounded operator such that $R(T) \subseteq D\left(A_{1}\right)=D(A)$. Let

$$
S_{1}=\frac{1}{2 \pi i} \int_{+B\left(D^{\prime}\right)} \frac{-1}{\gamma \lambda} R_{\lambda}^{\prime}\left(A_{1}\right) R_{1 / \lambda}^{\prime}(B) d \lambda
$$

and

$$
S_{2}=\frac{1}{2 \pi i} \int_{+B\left(D^{\prime}\right)} \frac{-1}{\gamma \lambda} R_{1 / \lambda}^{\prime}(B) R_{\lambda}^{\prime}\left(A_{1}\right) d \lambda
$$

Since $R\left(S_{1}\right) \subseteq D(A),(\gamma-\overline{B A}) S_{1}$ is defined, and $(\gamma-\overline{B A}) S_{1}=(\gamma-B A) S_{1}$.

$$
\begin{aligned}
\gamma-B A & =\gamma-B \gamma A_{1}=\gamma\left(I-B A_{1}\right) \\
& =\gamma B\left(\lambda-A_{1}\right)-\gamma \lambda B+\gamma I=\gamma B\left(\lambda-A_{1}\right)+\gamma(I-\lambda B) .
\end{aligned}
$$

Therefore,

$$
\begin{aligned}
(\gamma-\overline{B A}) S_{1}= & \frac{1}{2 \pi i} \int_{+B\left(D^{\prime}\right)} \frac{-1}{\lambda} B\left(\lambda-A_{1}\right) R_{\lambda}^{\prime}\left(A_{1}\right) R_{1 / \lambda}^{\prime}(B) d \lambda \\
& -\frac{1}{2 \pi i} \int_{+B\left(D^{\prime}\right)}(1 / \lambda-B) R_{\lambda}^{\prime}\left(A_{1}\right) R_{1 / \lambda}^{\prime}(B) d \lambda \\
= & \frac{1}{2 \pi i} \int_{+B\left(D^{\prime}\right)} \frac{-1}{\lambda} B\left[I+K_{1}(\lambda)\right] R_{1 / \lambda}^{\prime}(B) d \lambda \\
& -\frac{1}{2 \pi i} \int_{+B\left(D^{\prime}\right)}(1 / \lambda-B)\left[R_{1 / \lambda}^{\prime}(B) R_{\lambda}^{\prime}\left(A_{1}\right)+K_{2}(\lambda)\right] d \lambda \\
= & \frac{1}{2 \pi i} \int_{+B\left(D^{\prime}\right)} \frac{-1}{\lambda} B R_{1 / \lambda}^{\prime}(B) d \lambda+K_{3} \\
& -\frac{1}{2 \pi i} \int_{+B\left(D^{\prime}\right)}\left[I+K_{4}(\lambda)\right] R_{\lambda}^{\prime}\left(A_{1}\right) d \lambda+K_{5} \\
= & \frac{1}{2 \pi i} \int_{+B(D)} \frac{1}{\mu} B R_{\mu}^{\prime}(B) d \mu+K_{3}-\frac{1}{2 \pi i} \int_{+B\left(D^{\prime}\right)} R_{\lambda}^{\prime}\left(A_{1}\right) d \lambda+K_{6} .
\end{aligned}
$$


Since $0 \notin D$, we have

$$
\frac{1}{2 \pi i} \int_{+B(D)} \frac{1}{\mu} B R_{\mu}^{\prime}(B)-d \mu=B \frac{1}{2 \pi i} \int_{+B(D)} \frac{1}{\mu} R_{\mu}^{\prime}(B) d \mu=I+K_{7}
$$

by $[3$, Theorems 14,9 and $13, \S 2]$.

Since $R_{\lambda}^{\prime}\left(A_{1}\right)$ is analytic in $D^{\prime}$ except for at most a finite number of points, we have that $(2 \pi i)^{-1} \int_{+B\left(D^{\prime}\right)} R_{\lambda}^{\prime}\left(A_{1}\right) d \lambda=K_{8}$ by $[3$, Lemma $7.4, \S 2]$. Therefore, $(y-\overline{B A}) S_{1}=I+K^{\prime}, K^{\prime}, K_{i}, K_{i}(\lambda) \in K(X)$ for $i=1,2, \ldots, 8$.

Claim. $D(\overline{B A}) \subseteq D(A B)$ and $\overline{B A} x=A B x+K x \quad \forall x \in D(\overline{B A})$.

Proof. Let $x \in D(\overline{B A})$. Then there exists a sequence, $\left\{x_{n}\right\}_{n=1}^{\infty}$, such that $x_{n} \in D(A), x_{n} \rightarrow x$ as $n \rightarrow \infty$, and $B A x_{n} \rightarrow \overline{B A x}$ as $n \rightarrow \infty$. Therefore

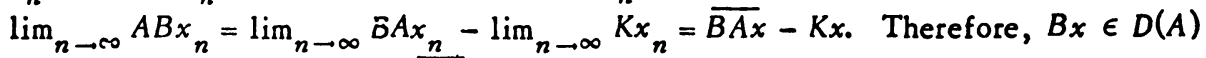
and $A B x=\lim _{n \rightarrow \infty} A B x_{n}=\frac{n}{B A x}-K x_{\text {. }}$

$$
\begin{aligned}
\gamma-\overline{B A}= & \overline{B\left(\lambda-A_{1}\right)}+\gamma(I-\lambda B)=\gamma\left(\lambda-A_{1}\right) B+\gamma(I-\lambda B)+K_{9} . \\
S_{2}(\gamma-\overline{B A})= & \frac{1}{2 \pi i} \int_{+B\left(D^{\prime}\right)} \frac{-1}{\gamma \lambda} R_{1 / \lambda}^{\prime}(B) R_{\lambda}^{\prime}\left(A_{1}\right) d \lambda\left[\gamma\left(\lambda-A_{1}\right) B+\gamma(I-\lambda B)+K_{9}\right] \\
= & \frac{1}{2 \pi i} \int_{+B\left(D^{\prime}\right)} \frac{-1}{\lambda} R_{1 / \lambda}^{\prime}(B)\left[I+K_{10}(\lambda)\right] B d \lambda \\
& +\frac{1}{2 \pi i} \int_{+B\left(D^{\prime}\right)} \frac{-1}{\gamma \lambda}\left[R_{\lambda}^{\prime}\left(A_{1}\right) R_{1 / \lambda}^{\prime}(B)-K_{2}(\lambda)\right]\left[\gamma(I-\lambda B)+K_{9}\right] d \lambda \\
= & {\left[\frac{1}{2 \pi i} \int_{+B(D)} \frac{1}{\mu} R_{\mu}^{\prime}(B) d \mu\right] B+K_{11} } \\
& +\frac{1}{2 \pi i} \int_{+B\left(D^{\prime}\right)}(-1) R_{\lambda}^{\prime}\left(A_{1}\right) R_{1 / \lambda}^{\prime}(B)(1 / \lambda-B) d \lambda+K_{12} \\
= & I+K_{13}+\frac{1}{2 \pi i} \int_{+B\left(D^{\prime}\right)}(-1) R_{\lambda}^{\prime}\left(A_{1}\right)\left[I+K_{14}(\lambda)\right] d \lambda \\
= & I+K_{15},
\end{aligned}
$$

$K_{i}, K_{i}(\lambda) \in K(x)$ for $i=9,10, \cdots, 14$.

Therefore $(\gamma-\overline{B A}) \in \Phi(X)$ by [2, Lemma 2.4]. This completes the proof that $\sigma_{\Phi}(\overline{B A}) \subseteq \sigma_{\phi}(B) \sigma_{\Phi}(A)$.

To show that $\sigma_{\Phi}(A B) \subseteq \sigma_{\Phi}(B) \sigma_{\Phi}(A)$ we proceed to show that $(\gamma-A B) \in \Phi(X)$. Let $S_{1}$ and $S_{2}$ be as above. Since $R\left(S_{1}\right) \subseteq D(A)$ and $A B x=B A x-K x, \forall x \in D(A)$, we have that $(\gamma-A B) S_{1}=(\gamma-B A) S_{1}+K_{15}=I+K^{\prime}+K_{15}=I+K_{16}$.

$$
\begin{aligned}
S_{2}(\gamma-A B) & =S_{2}\left[\gamma\left(\lambda-A_{1}\right) B+\gamma(I-\lambda B)\right] \\
& =I+K_{17}, \quad K_{15}, K_{16}, K_{17} \in K(X) .
\end{aligned}
$$

Therefore, by [2, Lemma 2.4], $(\gamma-A B) \in \Phi(X)$. This completes the proof that $\sigma_{\Phi}(A B) \subseteq \sigma_{\Phi}(B) \sigma_{\phi}(A)$. 
Corollary 2.1. Let $A \in C(X)$ and $B \in L(X)$. Assume $0 \notin \sigma(B)$. Let $B: D(A)$ $\rightarrow D(A)$ and let there exist a compact operator, $K$, sucb that $B A x=A B x+K x$, $\forall x \in D(A)$. Then $B A$ and $\left.A B\right|_{D(A)}$ are closed and

(1) $\sigma_{\phi}\left(\left.A B\right|_{D(A)}\right)=\sigma_{\phi}(B A) \subseteq \sigma_{\phi}(B) \sigma_{\phi}(A)$, and

(2) $\sigma_{\phi}(A B) \subseteq \sigma_{\phi}(B) \sigma_{\phi}(A)$.

Proof. Since $B$ is invertible and $A$ is closed, $B A$ is closed. We proceed to show that $\left.A B\right|_{D(A)}$ is closed.

Let $x_{n} \in D(A), x_{n} \rightarrow x$ and $A B x_{n} \rightarrow y$. Since $B$ is bounded and $A$ is closed, we have $y=A B x$. We have only to show that $x \in D(A)$. $B A x_{n}=\left(A B x_{n}+K x_{n}\right)$ converges to some vector, $z$. Therefore, $A x_{n} \rightarrow B^{-1} z$, and, since $A$ is closed, $x \in D(A)$. This shows that $\left.A B\right|_{D(A)}$ is closed.

Since $B A=\left.A B\right|_{D(A)}+K$, we have by $\left[2\right.$, Theorem 2.8] that $\sigma_{\phi}\left(\left.A B\right|_{D(A)}\right)=$ $\sigma_{\Phi}(B A)$. The remainder of the corollary follows from Theorem 2.

\section{BIBLIOGRAPHY}

1. Tosio Kato, Perturbation theory for nullity, deficiency and other quantities of linear operators, J. Analyse Math. 6 (1958), 261-322. MR 21 \#6541.

2. Martin Schechter, Basic theory of Fredholm operators, Ann. Scuola Norm. Sup. Pisa (3) 21 (1967), 261-280. MR 36 \#6977.

3. Jack Shapiro and Martin Schechter, A generalized operational calculus developed from Fredholm operator theory, Trans. Amer. Math. Soc. 175 (1973), 439-467.

4. A. E. Taylor, Spectral theory of closed distributive operators, Acta Math. 84 (1951), 189-224. MR 12, 717.

DEPARTMENT OF MATHEMATICS, BERNARD M. BARUCH COLLEGE (CUNY), NEW YORK, NEW YORK 10010

DEPARTMENT OF MATHEMATICS, QUEENS COLLEGE (CUNY), FLUSHING, NEW YORK 11367 